\title{
Qualidade do Posicionamento em Aplicativos VGI Obtido por Sensores de Localização em Smartphones
}

\author{
Positional Quality in VGI Apps Acquired by Smartphone's Location Sensors
}

\author{
Gabriel Araujo de Oliveira ${ }^{1}$ \\ José Almir Cirilo ${ }^{2}$ \\ Patricia Lustosa Brito ${ }^{3}$ \\ Elias Nasr Naim Elias ${ }^{4}$
}

Recebido em julho de 2019.

Aprovado em setembro de 2019.

\begin{abstract}
RESUMO
O artigo apresenta a avaliação da qualidade do posicionamento obtido por sensores de localização acoplados em smartphones, abordando o seu desempenho para a coleta de Informações Geográficas Voluntárias (VGI). Os experimentos foram realizados na forma de estudo de caso, em que as coordenadas coletadas em um aplicativo VGI (Hidromapp) foram comparadas com seus respectivos homólogos de referência. As amostras foram coletadas em condições distintas, utilizando 05 smartphones com características e configurações diferentes. Com base nas discrepâncias, a acurácia posicional foi calculada para cada uma das amostras através do indicador estatístico RMSE, incluindo a análise de diferentes fatores observados no experimento. Em seguida, a qualidade do posicionamento foi avaliada na perspectiva da destinação final dos dados (fitness-for-purpose), sendo observado se a acurácia posicional calculada atende aos propósitos do aplicativo Hidromapp. Foi identificado que smartphones possibilitam a realização de levantamentos com acurácia posicional planimétrica entre $5 \mathrm{~m}$ e 20m (faixa segura observada), dependendo das condições de levantamento, e das características e configurações dos dispositivos utilizados. Para o aplicativo Hidromapp, que visa fornecer dados observados de suporte para modelos hidrodinâmicos e simulação de inundações, o desempenho dos smartphones foi considerado satisfatório de acordo com o parecer dos pesquisadores consultados.
\end{abstract}

\footnotetext{
${ }^{1}$ Programa de Pós-graduação em Engenharia Civil, Universidade Federal de Pernambuco, Brasil. E-mail: goliveira.urb@gmail.com

${ }^{2}$ Programa de Pós-graduação em Engenharia Civil, Universidade Federal de Pernambuco, Brasil. E-mail: almir.cirilo@gmail.com

${ }^{3}$ Programa de Pós-graduação em Engenharia Civil, Universidade Federal da Bahia, Brasil.

E-mail: britopatricia@hotmail.com

${ }^{4}$ Programa de Pós-Graduação em Ciências Geodésicas, Universidade Federal do Paraná, Brasil.

E-mail: elias_naim2008@hotmail.com
} 
PALAVRAS-CHAVE: VGI. Smartphone. Acurácia Posicional. Hidromapp.

\begin{abstract}
The paper presents the positioning quality evaluation acquired by localization sensors coupled in smartphones, verifying its performance for Volunteered Geographic Information (VGI) field surveys. The experiments were carried as a case study, in which the coordinates collected in a VGI application (Hidromapp) were compared with their respective reference counterparts. The samples were collected under different conditions: using 05 smartphones with different characteristics and configurations. Based on the discrepancies, the positional accuracy was calculated for each of the samples, using the statistical indicator RMSE, including the analysis of different factors observed in the experiment. Then, the positioning quality was evaluated from the data use perspective (fitness-for-purpose), and was observed if the calculated positional accuracy is satisfactory to the Hidromapp application purposes. In results, it was identified that smartphones make possible spatial data collect with planimetric positional accuracy between $5 \mathrm{~m}$ and $20 \mathrm{~m}$ (safe range observed), depending on the survey conditions, and on the characteristics and configurations of the devices used. For the Hidromapp application, which aims to provide observed data support for hydrodynamic models and flood simulation, the performance of smartphones was considered satisfactory according to the researchers consulted.
\end{abstract}

KEYWORDS: VGI. Smartphone. Positional accuracy. Hidromapp.

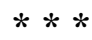

\title{
Introdução
}

$\mathrm{Na}$ última década, os sensores de localização acoplados em smartphones e tablets tem impulsionado, de forma mais intensa, o desenvolvimento de diversos aplicativos baseados em informações georreferenciadas (Waze, Uber, Ifood, CittaMobi, etc). Da mesma maneira, a crescente demanda por novos aplicativos tem incentivado o aprimoramento dos dispositivos móveis, criando assim novas perspectivas para as informações geográficas. Entre as mais diversas aplicações, os sistemas baseados em localização tornaram possível a aquisição de informações georreferenciadas produzidas por pessoas de todo o planeta, de forma colaborativa e independente do seu grau de instrução ou área de atuação, aqui entendidas como Volunteered Geographic Information (VGI), ou Informações Geográficas Voluntárias (GOODCHILD, 2007). 
Embora as plataformas VGI sejam hoje reconhecidas como uma importante fonte alternativa de dados espaciais, tendo o Open StreetMap como exemplo mais citado, a qualidade das informações é ainda uma questão a ser explorada (HAKLAY, 2010; ESMAIL, NAESERI e ESMAIL, 2013; BRAVO e SLUTER, 2015; HUNG, KALANTARI e RAJABIFARD, 2016; DÉ TRÉ et al., 2017; FONTE et al., 2017). Nesse contexto, as Ciências Geodésicas e Cartográficas, entre outras, têm sido acionadas para responder questões inerentes à aquisição de dados espaciais colaborativos e à sua respectiva qualidade.

Entre os atributos que compõem a qualidade de uma informação geográfica, a acurácia posicional pode ser considerada como a sua principal característica (FONTE et al., 2017; ISO 19157). A acurácia posicional planimétrica representa a capacidade de um equipamento, ou método, de estimar a posição (latitude e longitude) de um ponto na superfície terrestre, levando em consideração a presença de erros aleatórios (precisão) e de erros sistemáticos (tendências) nas medições (MONICO et al., 2009). Da mesma forma, o atributo também é um indicador da qualidade de um produto cartográfico, informando o quão discrepantes podem estar as feições mapeadas em relação às suas coordenadas de referência.

Logo, como os erros e incertezas estão presentes em todo e qualquer levantamento (MONICO et al., 2009), se torna fundamental avaliar a qualidade do posicionamento também em sistemas VGI. Nesta direção, os experimentos realizados até então estiveram concentrados na avaliação da acurácia posicional de sistemas VGI como o OpenStreetMap, em que as feições são vetorizadas a partir de imagens de satélite (HAKLAY, 2010; CRUZ e SANTOS, 2016; BROVELLI e ZAMBONI, 2018; ELIAS, 2019). Por sua vez, são escassos na literatura os trabalhos relacionados à acurácia posicional de dados espaciais colaborativos obtidos por meio de sensores de localização acoplados em smartphones.

É importante ressaltar que existem estudos relacionados à qualidade posicional dos sensores de localização em smartphones, chegando à resultados 
próximos ou até mesmo superiores aos receptores GNSS de navegação, como em Zandbergen (2009), Zandbergen e Barbeau (2011), Réquia (2013), Carrasco-Letelier (2015), Gonçalves e Borges (2016), Tomaštík et al. (2017), Hoffmann, Borelli e Schmidt Nanni (2018), Lucena (2018), entre outros. Porém, no caso de aplicativos VGI, e como proposto neste trabalho, é necessário considerar a diversidade de dispositivos e configurações utilizadas pelos usuários, as características do local mapeado, e principalmente, a inexistência de padrões metodológicos para a realização do levantamento pelos usuários.

Estes aspectos mencionados nem sempre são identificados nos trabalhos correlatos, ou são abordados de forma isolada. Além disso, os resultados são discutidos com enfoque para aplicações técnicas específicas, que não necessariamente retratam a realidade para aplicativos VGI.

Por tais questões, este trabalho se dedica exclusivamente à análise da qualidade do posicionamento obtido por sensores de localização em smartphones para aplicativos VGI. A abordagem aqui explorada não consiste em classificar as informações como "boas" ou "ruins" quando comparadas com produtos cartográficos de alta qualidade. Reconhece-se desde o princípio a relevância das plataformas VGI como fonte alternativa de dados espaciais, e busca-se então fornecer subsídios para qualificar sua coleta e utilização, no que depende especificamente do posicionamento planimétrico.

\section{Materiais e Métodos}

1.1 Área de estudo e pontos de verificação

O estudo foi realizado no Campus da Universidade Federal de Pernambuco (UFPE), localizado na cidade de Recife-PE, Brasil. A área possui alta densidade de Estações Rádio Base (ERB), configurando condições favoráveis para o posicionamento baseado em redes, incluindo A-GPS (TELEBRASIL). Com relação à presença de obstáculos físicos que podem interferir na qualidade do posicionamento, o campus possui características de 
áreas urbanas, contendo barreiras como edificações e árvores, e também, áreas abertas, com boas condições de levantamento.

O primeiro passo foi definir 50 pontos de verificação, em ortofotos com escala 1:1.000, distribuídos numa área de aproximadamente $0,3 \mathrm{~km}^{2}$, e cujas coordenadas foram utilizadas como referência na comparação com os dados coletados via dispositivos móveis. As ortofotos utilizadas são produtos cartográficos previamente validados e enquadrados no Padrão de Exatidão Cartográfica (PEC) com Classe A para a escala 1:1.000 (SILVA e NEVES, 2012).

Dessa forma, o produto apresenta incertezas posicionais de $0,28 \mathrm{~m}$, isto é, em uma ordem de grandeza superior à qualidade posicional atribuída à técnica de posicionamento absoluto (que é a utilizada nos levantamentos VGI cm smartphones). Sendo assim apropriada para a utilização como referência no estudo proposto.

Entre os 50 pontos de verificação, foram estabelecidos 25 pontos situados em locais com boas condições de levantamento (sem presença de obstáculos físicos), e 25 pontos em situações desfavoráveis (Figura 1).

Figura 1 - Pontos de verificação definidos na área de estudo

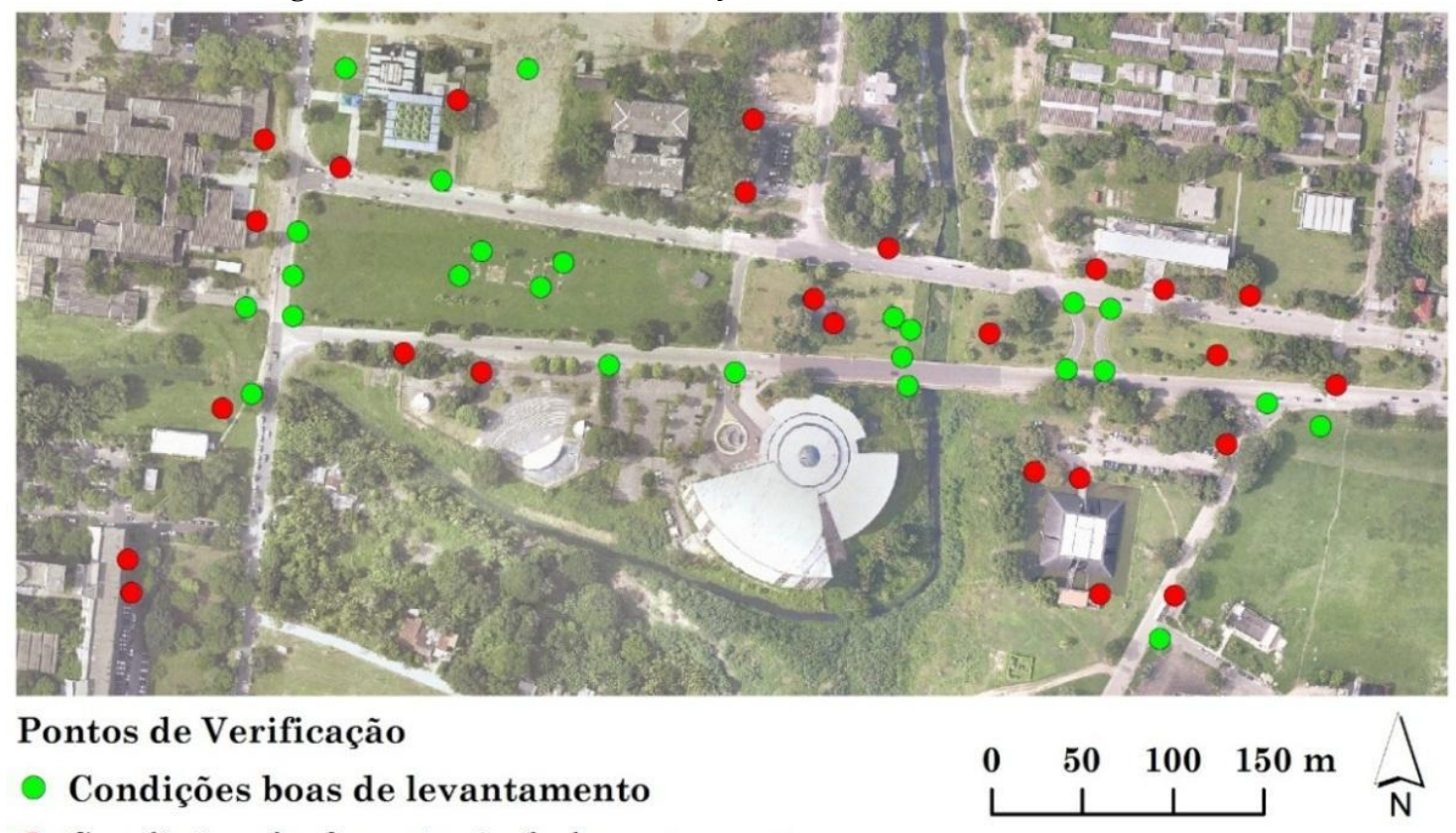

- Condições desfavoráveis de levantamento

Fonte: Elaborada pelos autores. 


\subsection{Smartphones Android}

Foram utilizados 05 smartphones Android, com diferentes modelos e configurações, considerando que a qualidade do posicionamento pode sofrer alterações de acordo com os recursos do dispositivo (ADUSEI, KYAMAKYA e JOBMANN, 2002; GUIMARÃES e GRIVET, 2003; ZEIMPEKIS et al., 2003; AKGUL e PAHLAVAN, 2009; ZANDBERGEN, 2009). Além disso, a coleta de dados por diferentes smartphones buscou simular a diversidade de dispositivos utilizados por usuários em sistemas VGI (Quadro 1).

Quadro 1 - Características e configurações dos dispositivos utilizados

\begin{tabular}{|c|c|c|c|c|c|}
\hline \multirow{2}{*}{$\begin{array}{c}\text { Características } \\
\text { Configuração }\end{array}$} & A & B & C & D & E \\
\cline { 2 - 6 } Marca / Modelo & $\begin{array}{c}\text { Asus } \\
\text { Zen Fone } 3\end{array}$ & $\begin{array}{c}\text { Asus } \\
\text { Zen Fone } 3\end{array}$ & $\begin{array}{c}\text { Multilaser } \\
\text { MS45S }\end{array}$ & $\begin{array}{c}\text { Samsung } \\
\text { Galaxy J5 }\end{array}$ & $\begin{array}{c}\text { Samsung } \\
\text { Galaxy S5 }\end{array}$ \\
\hline $\begin{array}{c}\text { Satélites } \\
\text { habilitados para } \\
\text { rastreio }\end{array}$ & $\begin{array}{c}\text { Glons, } \\
\text { Beidou }\end{array}$ & $\begin{array}{c}\text { Glons, } \\
\text { Beidou }\end{array}$ & Gps & $\begin{array}{c}\text { Glons, } \\
\text { Beidou }\end{array}$ & $\begin{array}{c}\text { Glons, } \\
\text { Modo de } \\
\text { Localização }\end{array}$ \\
\hline $\begin{array}{c}\text { Alta } \\
\text { Precisão }\end{array}$ & $\begin{array}{c}\text { Alta } \\
\text { Precisão }\end{array}$ & $\begin{array}{c}\text { Alta } \\
\text { Precisão }\end{array}$ & $\begin{array}{c}\text { Somente } \\
\text { Dispositivo }\end{array}$ & $\begin{array}{c}\text { Somente } \\
\text { Dispositivo }\end{array}$ \\
\hline A-GPS & Sim & Sim & Não & Sim & Sim \\
\hline Conexão 3G/4G & Sim & Não & Não & Sim & Não \\
\hline Conexão Wi-Fi & Não & Não & Não & Não & Não \\
\hline
\end{tabular}

Fonte: Elaborado pelos autores.

Entre os dispositivos, o Smartphone $C$ está enquadrado na categoria de desempenho inferior e o recurso A-GPS foi desabilitado para a coleta de dados, buscando verificar o comportamento de dispositivos com recursos tecnológicos e configurações de baixo desempenho.

O sistema operacional Android permite, na maioria das versões, que o usuário selecione o modo de localização utilizado para o posicionamento. $\mathrm{Na}$ prática, o modo "Somente dispositivo" obtém as coordenadas a partir de redes 
GNSS e/ou A-GPS; "Economia de bateria" considera apenas as informações provenientes de ERB's e/ou redes Wi-Fi (Cell-ID, Wi-Fi Mac-ID e A-GPS); e o modo "Alta precisão" utiliza todos os recursos citados, desde que disponíveis.

Em relação ao modo de localização, foram utilizados os modos "Alta Precisão" e "Somente Dispositivo". O modo "Economia de Bateria" foi descartado uma vez que a documentação do Sistema Operacional Android recomenda a sua utilização apenas para localização aproximada, com deslocamento superior à 100 metros (ANDROID DEVELOPERS, 2019).

No que se refere à conexão com redes de internet, nenhum dos dispositivos utilizou conexão de redes Wi-Fi (Wi-Fi Mac-ID), considerando que o trabalho busca avaliar o desempenho dos dispositivos para a realização de levantamentos outdoor.

\subsection{Aplicativo VGI (Hidromapp)}

O aplicativo utilizado para a coleta dos pontos em campo foi o Hidromapp (OLIVEIRA, BRITO e SANTOS, 2018; OLIVEIRA, CIRILO e RIBEIRO, 2018), que consiste numa aplicação mobile desenvolvida para o mapeamento colaborativo de registros de inundação (Figura 2). No aplicativo, o georreferenciamento das informações coletadas pode ser realizado de forma manual, utilizando consulta de endereços e mapa interativo ("apontar no mapa"), e em modo automático (“onde estou agora"), cujo posicionamento é obtido por meio dos sensores de localização. Neste trabalho, apenas o último método é abordado, buscando compreender a qualidade do posicionamento das informações coletadas utilizando sensores de localização acoplados em smartphones.

No modo de localização automática, o Hidromapp possui os recursos básicos dos sensores, e não utiliza algoritmos de otimização para o posicionamento. Dessa forma, ao coletar coordenadas utilizando apenas as funcionalidades básicas dos sensores, os resultados deste experimento podem ser aplicados à maioria dos aplicativos VGI. 
Figura 2 - Interface do aplicativo Hidromapp.

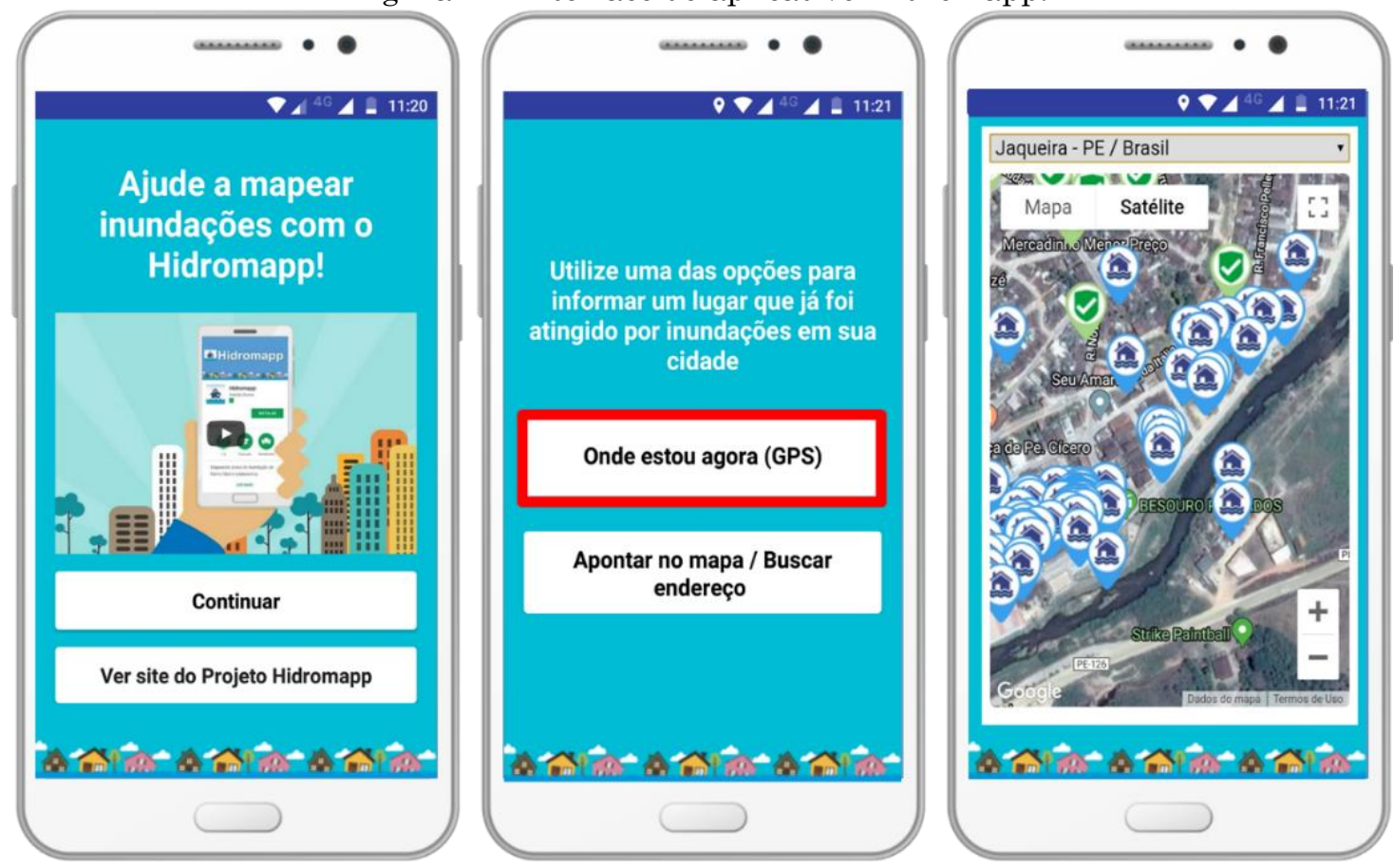

Fonte: Elaborada pelos autores.

Além das coordenadas e atributos relacionados à riscos hidrológicos, o aplicativo obtém informações como data e hora da coleta, ID do usuário, conexão do dispositivo (online ou offline), modo de localização utilizado pelo usuário, e a precisão estimada pelo sensor no momento da coleta (denominada accuracy em aplicativos Android).

\subsection{Levantamento de feições em campo}

Em campo, os pontos de controle foram visitados e a coleta foi realizada na mesma data e horário. Cada um dos pontos de verificação definidos $(n=50)$ foi levantado por todos os dispositivos (05), gerando assim um total de 250 pontos coletados para análise.

Embora seja recomendado que receptores GNSS estejam posicionados acima do corpo do operador durante a coleta, buscou-se simular a utilização dos smartphones de forma usual, uma vez que em aplicações de mapeamento colaborativo não se tem controle sobre os procedimentos utilizados pelos usuários (Figura 3). 
Figura 3 - Coleta de pontos em campo.
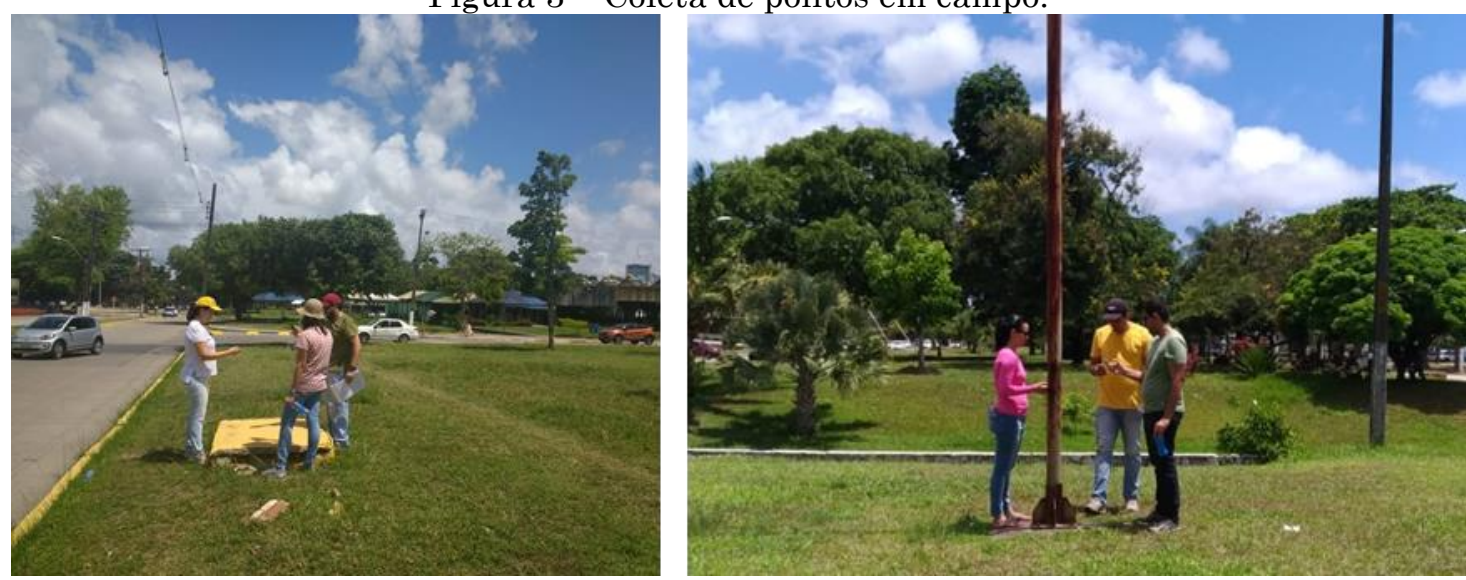

Fonte: Elaborada pelos autores.

Dessa forma, a análise da qualidade do posicionamento no trabalho considera a presença erros relacionados à interferência causada pelo corpo dos usuários, como naturalmente pode ser esperado em aplicações de mapeamento colaborativo via smartphone.

\subsection{Análise da acurácia posicional}

Após a coleta, os dados foram extraídos no banco de dados do Projeto Hidromapp, sendo realizada a conversão de coordenadas geográficas e Datum WGS 84, para a projeção UTM fuso 25S e Datum SIRGAS 2000. Os pontos foram removidos do banco de dados do projeto, já que não representam locais de inundação e foram coletados apenas para o experimento.

A acurácia posicional das amostras foi verificada de acordo com a abordagem conceitual apresentada Monico et al. (2009), sendo considerada a mensuração dos erros aleatórios (precisão) e efeitos sistemáticos (tendências). O cálculo da acurácia posicional foi realizado a partir da Raiz do Erro Médio Quadrático (Root Mean Square Error - RMSE). Como discutido em Monico et al. (2009) e Santos (2015), uma vez calculado a partir das discrepâncias posicionais, o RMSE é um indicador estatístico que representa a precisão e tendência dos dados num único valor, consistindo assim na sua acurácia. $\mathrm{O}$ indicador é recomendado para situações em que não é possível, ou necessário, mensurar as tendências de forma isolada. 
De acordo com Santos (2015) o cálculo do RMSE pode ser dado pela Equação 1:

$$
\operatorname{RMSE}=\sqrt{\frac{\sum\left(\mathrm{d}_{\mathrm{i}}\right)^{2}}{\mathrm{n}-1}}
$$

onde $d_{i}$ são as discrepâncias calculadas, $e$ é o número de pontos coletados.

O RMSE se constitui como um indicador adequado para o cálculo da acurácia posicional no trabalho, logo que, na maioria dos aplicativos, a coleta de dados por meio de sensores de localização não inclui pós-processamento para a correção de tendências ocasionadas por erros sistemáticos, principalmente quando se trata de sistemas VGI. Além do RMSE, também foram observadas as discrepâncias mínimas, máximas, média, desvio padrão, e outliers (método boxplot) calculadas para cada uma das amostras.

1.6 Análise da precisão fornecida pelo sensor do smartphone

De forma complementar, buscou-se verificar o comportamento da precisão estimada automaticamente pelos dispositivos. Em smartphones, ao obter as coordenadas de um ponto através dos sensores de localização, é retornado também o valor da precisão estimada pelo sensor, cujo método baseia-se no Erro Provável Circular, ou Circular Error Probability (LANGLEY, 1991; ADUSEI, KYAMAKYA e JOBMANN, 2002; GUIMARÃES e GRIVET, 2003).

Nas plataformas Android, a variável pode ser retornada através do método "getAccuracy()", e consiste no valor de um raio (dado em metros) em que existe $68 \%$ de probabilidade de que a posição verdadeira do dispositivo esteja inserida (ANDROID DEVELOPERS, 2019). Na prática, as coordenadas retornadas pelo sensor do smartphone consistem na média das observações realizadas durante o rastreio, e a variável accuracy representa o raio que contém $68 \%$ de todas as estimativas. Assim, a variável representa a precisão estimada (dispersão das observações), não incluindo as tendências, sobretudo 
porque as coordenadas de referência (posição verdadeira do dispositivo) são desconhecidas.

A análise foi realizada inicialmente a partir da taxa de acertos das estimativas dos sensores, observando se a posição verdadeira dos dispositivos estava inserida no raio estimado. Como exemplo, a Figura 4 ilustra uma inconsistência (erro) na precisão estimada, logo que o ponto verificação (B) não está inserido no raio de precisão dado pelo sensor (C).

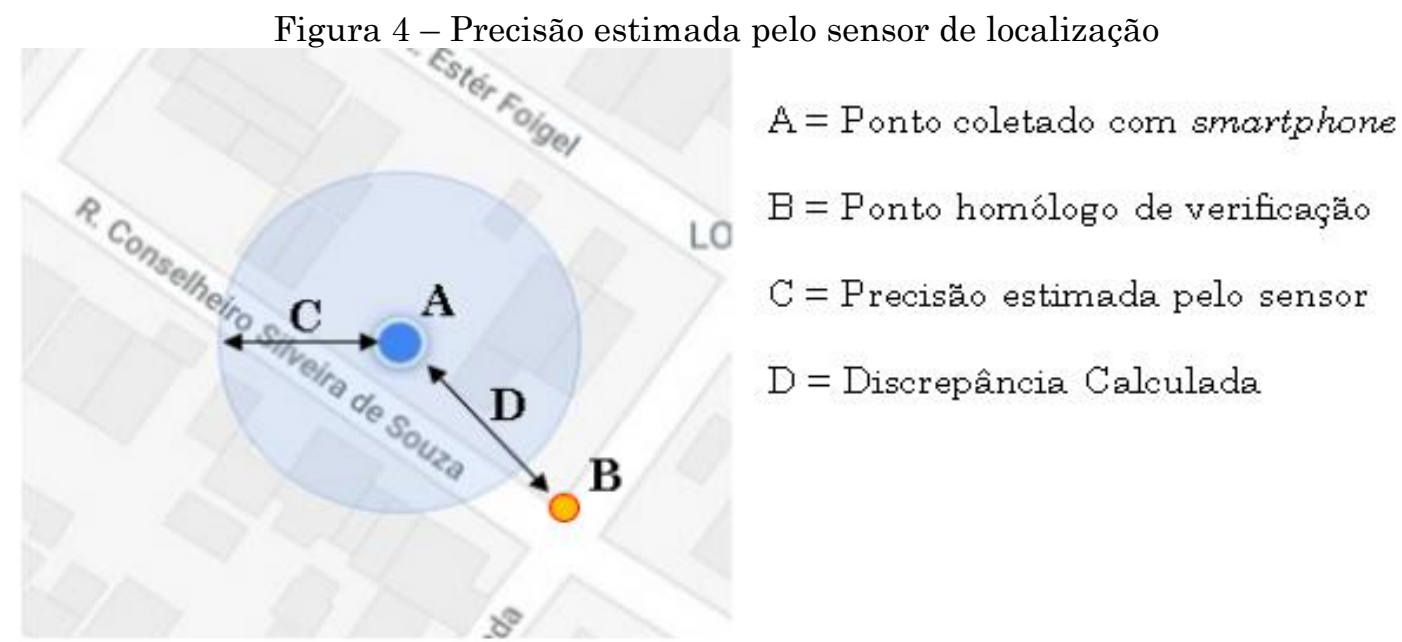

Fonte: Elaborada pelos autores.

Além disso foi observado grau de associação entre a precisão estimada pelos sensores e as discrepâncias calculadas, através dos coeficientes $R^{2} \mathrm{e}$ Pearson. Tomando a Figura 4 novamente como exemplo, buscou-se identificar as relações entre "C" (precisão estimada pelo dispositivo) e "D" (discrepâncias calculadas).

\subsection{Avaliação da qualidade do posicionamento}

Em trabalhos relacionados à qualidade posicional em VGI, é possível observar diferentes pontos de vista, e que se dividem entre questionamentos sobre a sua aplicação no sentido estrito das Ciências Geodésicas e Cartográficas, e aqueles que atestam a sua qualidade para determinados fins. Nesse contexto, com o reconhecimento dos sistemas VGI enquanto fonte alternativa de dados espaciais, as pesquisas decorrentes passaram a abordar 
a qualidade dos dados na perspectiva da sua destinação final (fitness-for-use, fitness-for-purpose, usability), como mencionado em Grira, Bédard e Roche (2010), Haklay (2010), Brown et al. (2013), Barron, Neis e Zipf (2014), Antoniou e Skopeliti (2015), Fonte et al. (2017), Degrossi et al. (2018).

Congregando com esta abordagem, é possível afirmar que a qualidade do posicionamento pode ter diferentes avaliações em função da destinação final dos dados, ainda que a sua acurácia posicional seja a mesma. Na prática, este tratamento já é utilizado para receptores GNSS de navegação, os quais são recomendados para determinados fins, mesmo apresentando acurácia posicional consideravelmente inferior aos receptores de alto desempenho.

Por isso, a avaliação da qualidade do posicionamento neste trabalho foi realizada em função da destinação final das informações coletadas. Também por esta razão, optou-se por utilizar apenas o RMSE e não o PEC-PCD (destinado à avaliação da qualidade de cartas de base para múltiplos fins). Enfatiza-se que neste experimento, apenas a acurácia planimétrica está sendo avaliada, e não se considera outros atributos de qualidade, como acurácia temática, completude, qualidade temporal e consistência lógica.

Como apresentado em Oliveira, Cirilo e Ribeiro (2018), o aplicativo Hidromapp tem como propósito reunir informações colaborativas sobre inundações já ocorridas, servindo como dados de suporte em modelos hidrodinâmicos e mapeamento de inundações, sobretudo na calibração e validação das simulações. Assim, por meio de entrevistas, foram consultados pesquisadores em mapeamento de riscos hidrológicos e modelagem hidrodinâmica, com o intuito de avaliarem a acurácia posicional calculada em função da sua aplicabilidade, especificamente como dados de suporte em simulação de inundações.

Ao todo, foram entrevistados 03 professores-pesquisadores, com no mínimo 20 anos de experiência no tema abordado, e 05 pesquisadores de PósGraduação que atualmente estão realizando pesquisas com aplicação de modelos hidrodinâmicos para simulação de inundações. 


\section{Resultados e Discussão}

\subsection{Acurácia posicional planimétrica calculada}

Desde o princípio, foi identificada a interferência das condições de levantamento no desempenho dos dispositivos. Em todos os casos, o RMSE calculado foi superior para as coordenadas coletadas em locais com presença de obstáculos físicos (Tabela 1 e Figura 5).

Tabela 1 - Acurácia calculada para as amostras coletadas (RMSE)

\begin{tabular}{c|c|c|c}
\hline \multirow{2}{*}{ Dispositivos } & \multicolumn{2}{|c|}{ Condições de Levantamento } & \multirow{2}{*}{ Todos os pontos } \\
\cline { 2 - 3 } & Boas $(\mathbf{n = 2 5 )}$ & Ruins (n=25) & \\
\hline Smartphone A & $8,9 \mathrm{~m}$ & $10,8 \mathrm{~m}$ & $9,8 \mathrm{~m}$ \\
\hline Smartphone B & $11,9 \mathrm{~m}$ & $20,4 \mathrm{~m}$ & $16,6 \mathrm{~m}$ \\
\hline Smartphone C & $22,5 \mathrm{~m}$ & $40,4 \mathrm{~m}$ & $32,4 \mathrm{~m}$ \\
\hline Smartphone D & $5,2 \mathrm{~m}$ & $7,6 \mathrm{~m}$ & $6,5 \mathrm{~m}$ \\
\hline Smartphone E & $11,7 \mathrm{~m}$ & $13,4 \mathrm{~m}$ & $12,5 \mathrm{~m}$ \\
\hline
\end{tabular}

Fonte: Elaborada pelos autores.

Figura 5 - Discrepâncias calculadas por smartphone e condição de levantamento

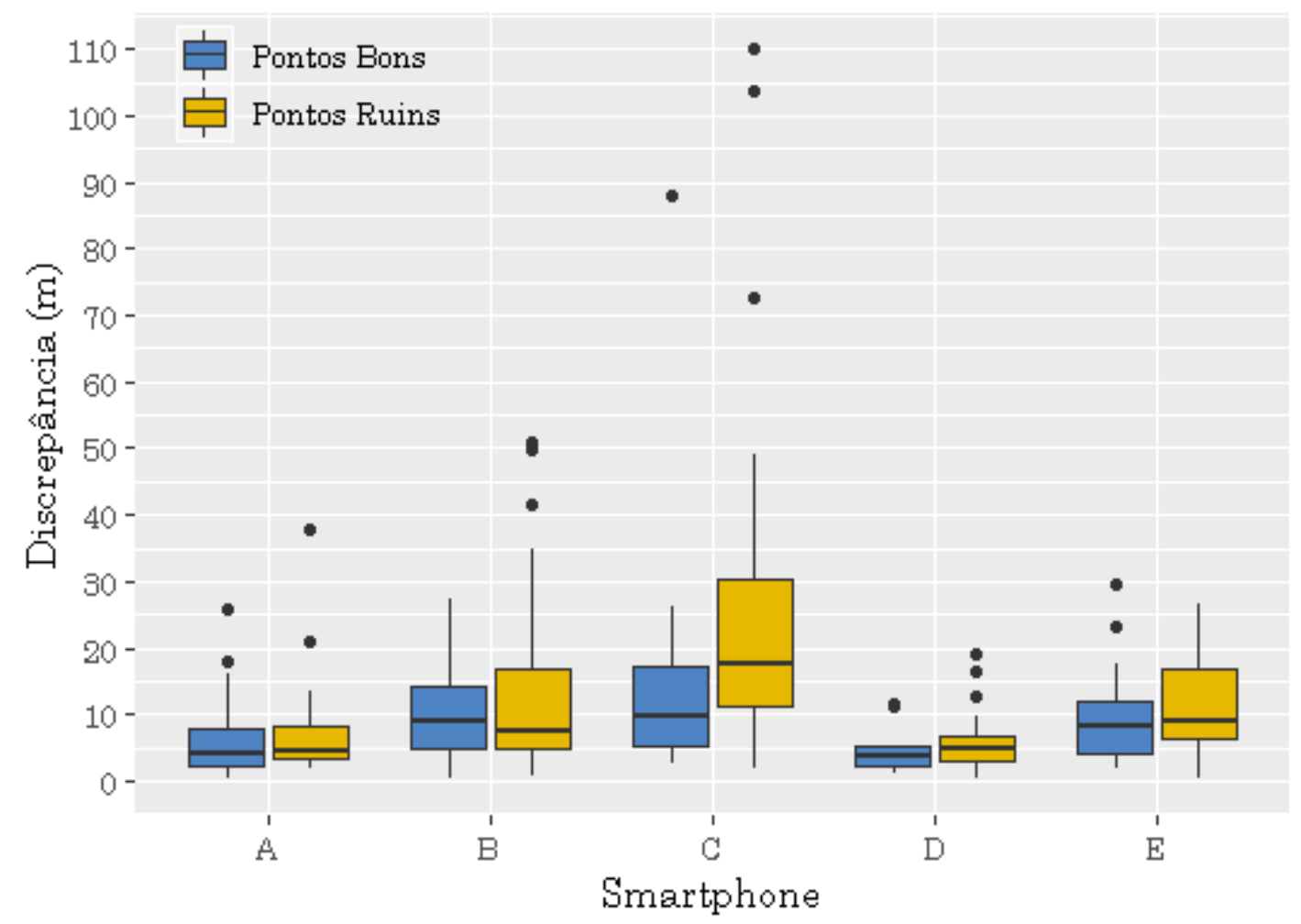

Fonte: Elaborada pelos autores. 
Também foi observado desempenho superior das amostras coletas por smartphones com conexão de redes $3 \mathrm{G} / 4 \mathrm{G}$, em comparação com os dispositivos offline. Esta constatação pode ser evidenciada no melhor desempenho obtido pelo dispositivo A (online) em relação ao smartphone B (offline), sendo que ambos compartilhavam das mesmas características e configurações, com exceção da conectividade 3G/4G (Tabela 1 e Figura 5).

Estes resultados corroboram com as observações mencionadas por Adusei, Kyamakya e Jobmann (2002), Akgul e Pahlavan (2009) e Zandbergen (2009). De acordo com os autores, e como verificado neste experimento, a acurácia posicional sofre interferência direta das condições de levantamento, podendo ser ainda inferior para a realização de levantamentos em ambientes internos (indoor). Por outro lado, também é mencionado que a utilização de redes Wi-Fi, em conjunto (soluções híbridas), pode melhorar o posicionamento pelos sensores, demandando assim novos experimentos incluindo levantamento indoor e com posicionamento apoiado em redes Wi-Fi.

Em relação ao desempenho individual dos dispositivos, o smartphone $\mathrm{C}$ obteve desempenho consideravelmente inferior em comparação com os demais aparelhos. Enquanto que o RMSE para os smartphones A, B, D e E oscilou entre $5 \mathrm{~m}$ e $12 \mathrm{~m}$ para os pontos em boas condições de levantamento, as amostras coletadas com o dispositivo C resultaram no RMSE de 22m. Para os pontos levantados em condições ruins, a acurácia calculada para o smartphone $\mathrm{C}$ foi de $40,4 \mathrm{~m}$, correspondendo ao dobro do maior valor obtido nos demais dispositivos, que variou entre $7,6 \mathrm{~m}$ e $20,4 \mathrm{~m}$.

$\mathrm{O}$ mesmo foi observado para o smartphone $\mathrm{C}$ em relação às demais estatísticas calculadas, em que as discrepâncias mínimas, máximas e médias, assim como o desvio padrão, apresentaram desempenho inferior comparado com os outros dispositivos. Na Figura 5 é possível observar que os outliers (boxplot) detectados nas amostras coletadas com o smartphone $\mathrm{C}$ são consideravelmente superiores, chegando a discrepâncias acima de $100 \mathrm{~m}$.

O recurso A-GPS é mencionado na literatura como o principal componente do posicionamento em smartphones, levando ao entendimento de 
que a não utilização do recurso no Smartphone C acarretou nas elevadas discrepâncias e baixa acurácia posicional. Porém, também é necessário incluir como fator o conjunto de hardware e software do dispositivo, que é inferior aos demais smartphones utilizados.

Neste ponto, é necessário mencionar que atualmente apenas em situações incomuns serão encontrados smartphones que permitem desativar o recurso A-GPS através das configurações do sistema operacional. Assim, esta é uma situação que pode ser considerada atípica em aplicativos VGI. Como recomendação, sugere-se que plataformas VGI restrinjam a coleta de dados em dispositivos com recurso A-GPS desativado, ou que esta informação conste entre os metadados das colaborações.

Por sua vez, o Smartphone D apresentou o melhor resultado, com acurácia posicional de aproximadamente $5,2 \mathrm{~m}$ e $7,6 \mathrm{~m}$ para os pontos levantados em condições boas e ruins respectivamente (Tabela 1 e Figura 5). O dispositivo também obteve melhor desempenho para os demais indicadores estatísticos (discrepância mínima, máxima, média, desvio padrão, e outliers).

Por fim, os dispositivos B e E tiveram desempenho intermediário para os pontos levantados em condições boas, com RMSE de 11,9m e 11,7m respectivamente. Já para os pontos em condições ruins, o smartphone $\mathrm{B}$ demonstrou maior sensibilidade, com RMSE de 20,4m, enquanto que o valor obtido para o dispositivo $\mathrm{E}$ foi $13,4 \mathrm{~m}$ para esta condição de levantamento (Tabela 1 e Figura 5)

Foi observado que o modo de localização "Alta precisão", utilizado apenas nos dispositivos A, B e C, não necessariamente apresentou acurácia posicional superior ao modo "Somente dispositivo", principalmente porque não foram utilizadas redes Wi-Fi. Assim, além das condições de levantamento, a utilização de diferentes dispositivos e configurações também contribuiu para a heterogeneidade da acurácia posicional.

Neste sentido, a lista de provedores de localização (location providers) e conexão do dispositivo (3G/4G/Wi-Fi) utilizados para o levantamento é um importante metadado em plataformas VGI, auxiliando no processo de 
avaliação da qualidade do posicionamento. Porém, é necessário que sejam realizados experimentos específicos para o estabelecimento das relações entre o erro planimétrico esperado e as características dos dispositivos.

Em comparação com trabalhos correlatos (Tabela 2), os resultados dos dispositivos $\mathrm{A}, \mathrm{D}$ e $\mathrm{E}$ foram compatíveis, enquanto que o smartphone $\mathrm{B}$ apresentou valores acima para os pontos levantados em condições ruins $(R M S E=20,4 \mathrm{~m})$. Por sua vez, o Smartphone $\mathrm{C}$ obteve desempenho consideravelmente inferior, demonstrando novamente a importância do recurso A-GPS para o posicionamento em smartphones.

Tabela 2 - Desempenho do posicionamento de smartphones em trabalhos correlatos

\begin{tabular}{|c|c|c|c|c|}
\hline Referência & Indicador & $\begin{array}{c}\text { Desempenho } \\
\text { posicional } \\
\text { planimétrico }\end{array}$ & $\begin{array}{c}\text { Local de } \\
\text { Levantamento }\end{array}$ & País \\
\hline $\begin{array}{l}\text { Hoffmann, } \\
\text { Borelli e Schmidt } \\
\text { Nanni (2018) }\end{array}$ & $\begin{array}{l}\text { Discrepância } \\
\text { Média }\end{array}$ & $6 \mathrm{~m}$ & $\begin{array}{l}\text { Área rural, Outdoor, } \\
\text { Condições não } \\
\text { informadas }\end{array}$ & Brasil \\
\hline \multirow{3}{*}{$\begin{array}{l}\text { Tomaštík et al. } \\
\text { (2017) }\end{array}$} & \multirow{3}{*}{ RMSE } & $1,9 \mathrm{~m}-2,4 \mathrm{~m}$ & $\begin{array}{l}\text { Área rural, Outdoor, } \\
\text { Condições boas }\end{array}$ & \multirow{3}{*}{ Eslováquia } \\
\hline & & $5 \mathrm{~m}-6,7 \mathrm{~m}$ & $\begin{array}{l}\text { Área rural, Outdoor, } \\
\text { Condições } \\
\text { intermediárias }\end{array}$ & \\
\hline & & $6,7 \mathrm{~m}-11,45 \mathrm{~m}$ & $\begin{array}{l}\text { Área rural, Outdoor, } \\
\text { Condições ruins }\end{array}$ & \\
\hline $\begin{array}{c}\text { Carrasco-Letelier } \\
\text { (2015) }\end{array}$ & $\begin{array}{l}\text { Discrepância } \\
\text { Média }\end{array}$ & $4,5 \mathrm{~m}$ & Não informado & Uruguai \\
\hline Réquia (2013) & $\begin{array}{l}\text { Discrepância } \\
\text { Média }\end{array}$ & $2,7 \mathrm{~m}-6,5 \mathrm{~m}$ & $\begin{array}{l}\text { Área urbana, Outdoor, } \\
\text { Condições boas }\end{array}$ & Brasil \\
\hline $\begin{array}{l}\text { Zandbergen } \\
\quad(2009)\end{array}$ & RMSE & $9 \mathrm{~m}$ & $\begin{array}{l}\text { Área urbana, Outdoor, } \\
\text { Condições boas }\end{array}$ & México \\
\hline
\end{tabular}

Fonte: Elaborada pelos autores.

Ainda em comparação com trabalhos correlatos, foi possível observar uma maior heterogeneidade no desempenho dos dispositivos utilizados neste experimento. Assim, pode-se afirmar que os erros posicionais planimétricos para smartphones podem oscilar predominantemente entre 5 e 20 metros, ou até mesmo com acurácia inferior em situações atípicas (Smartphone C). 
2.2 Precisão estimada pelo dispositivo

Apenas os smartphones A e D apresentaram taxa de acertos significativa, em que a as coordenadas de referência estavam inseridas no raio estimado pelo dispositivo (Tabela 3). Para os demais smartphones, a taxa de acertos esteve abaixo de $52 \%$, chegando à $20 \%$ nos pontos coletados com o Smartphone C em condições ruins de levantamento (Tabela 3).

Em relação ao grau de associação entre precisão estimada e as discrepâncias calculadas, não foram encontrados valores significantes para os coeficientes $R^{2}$ e Pearson (Tabela 3 e Figura 6). Em todas as amostras, os valores de ambos os indicadores estiveram aproximadamente entre 0,0 e 0,5, correspondendo à inexistência de correlação entre as variáveis.

Tabela 3 - Desempenho da precisão estimada - índices de acerto e correlação

\begin{tabular}{c|c|c|c|c|c|c|c|c|c|c}
\hline & \multicolumn{2}{|c|}{ Smartphone A } & \multicolumn{2}{c|}{ Smartphone B } & \multicolumn{2}{c}{ Smartphone C } & \multicolumn{2}{c}{ Smartphone D } & \multicolumn{2}{c}{ Smartphone E } \\
\cline { 2 - 11 } & $\begin{array}{c}\text { Pontos } \\
\text { Bons }\end{array}$ & $\begin{array}{c}\text { Pontos } \\
\text { Ruins }\end{array}$ & $\begin{array}{c}\text { Pontos } \\
\text { Bons }\end{array}$ & $\begin{array}{c}\text { Pontos } \\
\text { Ruins }\end{array}$ & $\begin{array}{c}\text { Pontos } \\
\text { Bons }\end{array}$ & $\begin{array}{c}\text { Pontos } \\
\text { Ruins }\end{array}$ & $\begin{array}{c}\text { Pontos } \\
\text { Bons }\end{array}$ & $\begin{array}{c}\text { Pontos } \\
\text { Ruins }\end{array}$ & $\begin{array}{c}\text { Pontos } \\
\text { Bons }\end{array}$ & $\begin{array}{c}\text { Pontos } \\
\text { Ruins }\end{array}$ \\
\hline $\begin{array}{c}\text { Taxa de } \\
\text { Acertos }\end{array}$ & $72 \%$ & $68 \%$ & $36 \%$ & $52 \%$ & $48 \%$ & $20 \%$ & $92 \%$ & $80 \%$ & $36 \%$ & $28 \%$ \\
\hline Pearson & 0,5 & 0,43 & 0,08 & 0,31 & 0,21 & 0,35 & $-0,11$ & 0,25 & 0,53 & 0,48 \\
\hline$R^{2}$ & 0,22 & 0,15 & $-0,04$ & 0,06 & 0,001 & 0,08 & $-0,03$ & 0,02 & 0,24 & 0,19 \\
\hline
\end{tabular}

Fonte: Elaborada pelos autores.

Além da inexistência de correlação entre os dados quando analisados em conjunto, também foram observadas inconsistências na análise individual das informações. Em alguns casos a discrepância calculada esteve acima de 20 metros, enquanto que a respectiva precisão foi estimada em 5 metros. Da mesma forma, foram detectadas situações inversas, em que o ponto apresentou discrepância calculada de 3,5m e precisão estimada em 40m.

Estas situações, entre outras, podem ser observadas na Figura 6, e permitem afirmar que a precisão estimada pelos sensores não possibilitou, neste experimento, a sua aplicação como indicador de qualidade do posicionamento dos dados. Tais observações congregam com os resultados 
obtidos por Tomaštík et al. (2017), em que também não foram detectadas relações significativas entre as variáveis.

Figura 6 - Desempenho da precisão estimada (accuracy) - gráficos de correlação
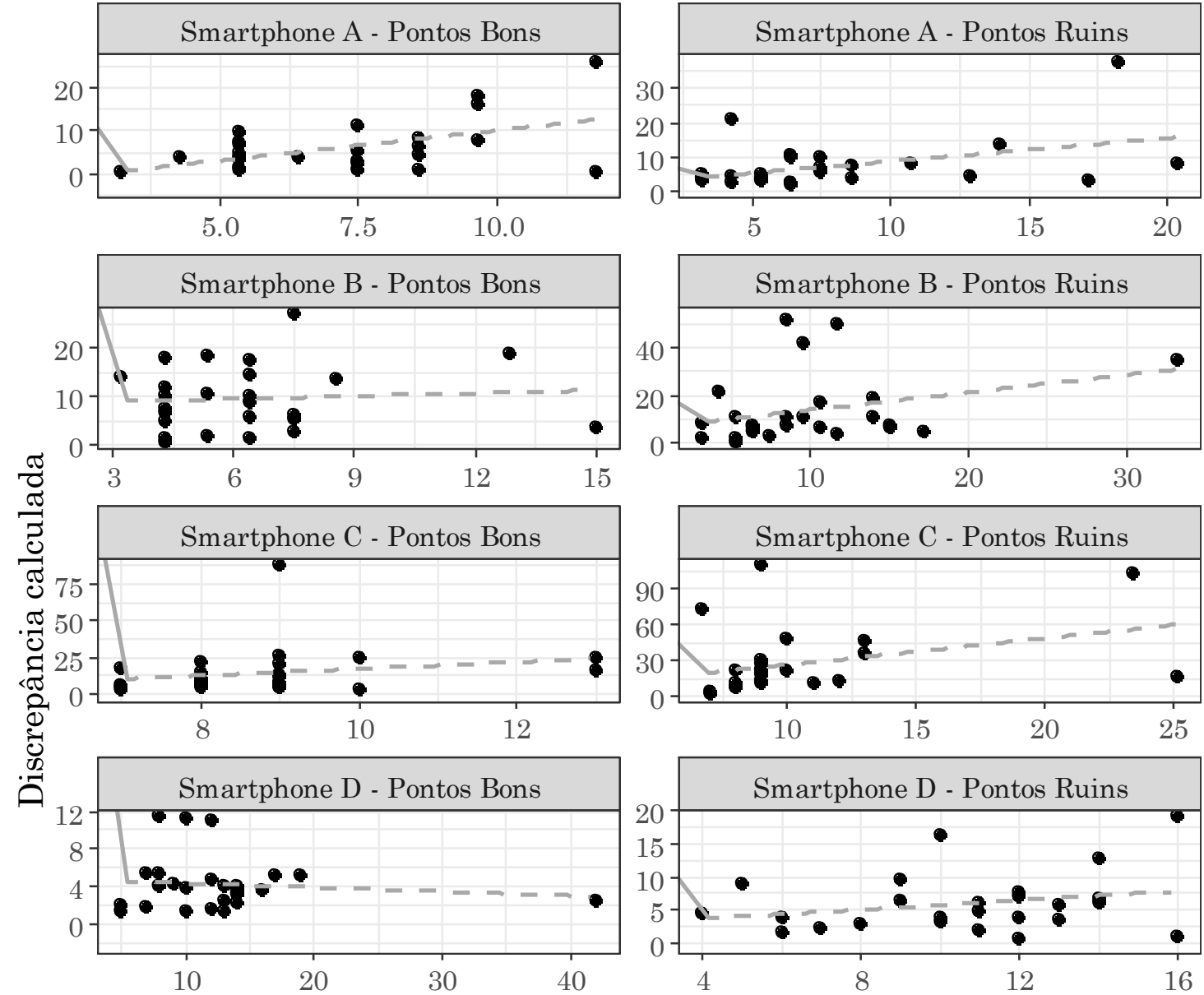
2.3 Qualidade do posicionamento quanto ao uso dos dados

De acordo com os pesquisadores consultados, dados observados (marcas de cheia) coletados com acurácia posicional planimétrica de até 15 metros, em geral, são adequados para a calibração e validação de modelos hidrodinâmicos e simulação de inundações, com algumas ressalvas para a sua utilização em situações específicas.

Como fatores para a avaliação, os entrevistados mencionaram que a indisponibilidade de dados de suporte para modelos hidrodinâmicos já tem acarretado na utilização de informações consideradas de média e baixa acurácia posicional planimétrica, como: modelos digitais de terreno com baixa resolução espacial (SRTM); marcas de cheia coletadas com receptores GNSS de navegação (acurácia planimétrica compatível com smartphones), ou até mesmo fotografias de inundações já ocorridas. Além disso, muitas vezes não é possível realizar a calibração e validação de modelos hidrodinâmicos devido à escassez de dados observados, de forma que os entrevistados salientaram que toda informação é útil, desde que a margem de erro seja conhecida.

De acordo com os pesquisadores, é preciso considerar as características do terreno para a utilização das informações. Observando que em modelos hidrodinâmicos as discrepâncias planimétricas poderão acarretar em erros na estimativa da profundidade da inundação, áreas planas permitem a aplicação de dados com acurácia posicional inferior, onde as cotas altimétricas sofrem pequenas variações. Por sua vez, os erros acarretados pela acurácia posicional planimétrica podem ser maiores em terrenos com alta declividade.

Assim, para os entrevistados, numa escala de qualidade para aplicação, a acurácia posicional planimétrica pode ser classificada em: a) ótimo, se até 5 metros; b) bom, se até 10 metros; e c) satisfatória, se até 15 metros, ou até 20 metros em determinados casos. Erros planimétricos acima de 20 metros foram considerados inadequados para utilização, podendo ser utilizado em locais de baixa declividade, onde a profundidade da inundação pode ser considerada a mesma, independente do deslocamento do ponto (marca de cheia). 
Em relação aos erros planimétricos identificados no trabalho, apenas o Smartphone $\mathrm{C}$ apresentou acurácia posicional inferior a 20 metros, enquanto que os demais dispositivos se enquadraram nos limites de tolerância definidos pelos pesquisadores. O Smartphone $D$ obteve o melhor desempenho, considerando: a sua acurácia posicional calculada de $6,5 \mathrm{~m}$, que $70 \%$ dados coletados apresentaram discrepância de até 5 metros, e que aproximadamente $90 \%$ dos pontos resultaram em erros planimétricos abaixo de 10 metros (Tabela 4). O Smartphone A foi classificado como bom desempenho, com erro planimétrico por volta de $10 \mathrm{~m}$, e os dispositivos B e E estiveram em posição intermediária, com erro planimétrico de até 15 metros.

Tabela 4. Percentual de pontos identificados por intervalo de erro planimétrico

\begin{tabular}{l|c|c|c|c|c}
\hline Smartphone & Até 5m & Até 10m & Até 15m & Até 20m & Acima de 20m \\
\hline Smartphone A & $58 \%$ & $84 \%$ & $90 \%$ & $94 \%$ & $6 \%$ \\
\hline Smartphone B & $30 \%$ & $60 \%$ & $76 \%$ & $88 \%$ & $12 \%$ \\
\hline Smartphone C & $20 \%$ & $38 \%$ & $56 \%$ & $66 \%$ & $34 \%$ \\
\hline Smartphone D & $70 \%$ & $88 \%$ & $96 \%$ & $100 \%$ & $0 \%$ \\
\hline Smartphone E & $28 \%$ & $66 \%$ & $80 \%$ & $90 \%$ & $10 \%$ \\
\hline
\end{tabular}

Assim, a acurácia posicional planimétrica identificada no trabalho foi considerada adequada quanto ao uso, uma vez que erro planimétrico identificado no aplicativo Hidromapp enquadrou-se nos limites estipulados, e sendo também compatível com os dados já utilizados atualmente. Por se tratar de modelos computacionais para a simulação de fenômenos naturais, os entrevistados mencionaram que os erros são inevitáveis, e que constantemente são adotados procedimentos para a sua minimização. Nesse sentido, os entrevistados apresentaram a existência de mecanismos para tratamento das informações, concluindo que os dados podem ser utilizados sem o comprometimento dos resultados. Em todos os casos, foi recomendada a utilização de informações em quantidade e distribuição espacial adequada para análise de consistência e minimização dos erros. 


\section{Conclusões}

Com base na avaliação da acurácia posicional planimétrica realizada neste trabalho, foi identificado que o desempenho obtido com o aplicativo VGI Hidromapp apresentou qualidade satisfatória para o seu propósito.

Considerando também os resultados de trabalhos correlatos, foi identificado que sensores de localização acoplados em smartphones apresentam acurácia posicional planimétrica adequada para aplicações compatíveis com receptores GNSS de navegação. Corroborando ainda com trabalhos correlatos, verificou-se que a acurácia posicional planimétrica em smartphones pode sofrer interferência das condições de levantamento, como a presença de obstáculos físicos, e das características e configurações dos dispositivos utilizados.

Como resultados inéditos alcançados neste trabalho, a acurácia posicional planimétrica identificada encontra-se numa realidade mais próxima para aplicações VGI, considerando a utilização de aplicativos que possuem recursos básicos de posicionamento, e situações em que o usuário não adota todos procedimentos necessários para a realização do levantamento. Em comparação com estudos correlatos, foi identificada uma maior heterogeneidade entre os smartphones utilizados neste trabalho, extrapolando a acurácia planimétrica obtida pelos demais autores.

Também foi possível mensurar a redução drástica ocasionada na acurácia quando o recurso A-GPS se encontra indisponível durante o levantamento, com desempenho consideravelmente inferior em comparação com os demais dispositivos utilizados. Porém, é necessário salientar que esta é uma situação incomum, e que neste estudo o Smartphone $C$ foi configurado propositalmente para operar sem o recurso A-GPS.

Para aplicativos VGI, observou-se que as características do dispositivo e sua respectiva configuração no momento do posicionamento podem ser metadados importantes para estimar da qualidade do posicionamento, embora não tenham sido explorados neste trabalho. Por outro lado, em relação 
à precisão estimada automaticamente pelos sensores (accuracy), que é um metadado de fácil aquisição em aplicativos móveis, não foi identificada correlação quando comparada com as discrepâncias calculadas para a maioria das amostras.

Por fim, a avaliação da qualidade do posicionamento na perspectiva da sua destinação final permitiu a análise adequada das informações, sendo recomendada para situações em que os dados coletados não necessariamente devem apresentar acurácia posicional compatível com levantamentos de alto desempenho.

\section{Agradecimentos}

Os autores agradecem à Fundação de Amparo à Ciência e Tecnologia de Pernambuco (FACEPE) pelo financiamento da pesquisa por meio de bolsa de Pós-Graduação (Processo IBPG-0051-3.01/17), e aos membros do Grupo de Pesquisa em Recursos Hídricos (GRH/UFPE) que contribuíram na realização dos levantamentos em campo.

\section{Contribuição dos autores}

Gabriel Araujo de Oliveira: Doutorando responsável pela pesquisa, desenvolvedor do aplicativo Hidromapp, contribuiu na estruturação do escopo do artigo, coleta e sistematização de dados, análise dos resultados, redação e revisão do artigo.

Prof. Dr. José Almir Cirilo: Orientador da pesquisa, contribuiu na estruturação do escopo do estudo, supervisão dos procedimentos metodológicos, análise dos resultados, e revisão do manuscrito.

Prof $^{\mathrm{a}}$. Dr ${ }^{\mathrm{a}}$. Patricia Lustosa Brito: Contribuiu na estruturação do escopo do artigo, análise dos resultados, redação e revisão do manuscrito.

Elias Nasr Naim Elias: Contribuiu na estruturação dos procedimentos metodológicos, sistematização de dados, análise dos resultados, redação e revisão do manuscrito. 


\section{Referências}

ADUSEI, I. K.; KYAMAKYA, K.; JOBMANN, K. Mobile positioning technologies in cellular networks: An evaluation of their performance metrics. Proceedings of 21st Military Communications Conference, Anaheim, 2002. pp. 12391244.

AKGUL, F. O.; PAHLAVAN, K. Location awareness for everyday smart computing. Proceedings of 10th International Conference on Telecommunications, Zagreb, 2009. pp 2-7.

ANDROID DEVELOPERS. Site

<https://developer.android.com/reference/android/location/Location.html>, acessado em 03 de abril de 2019.

ANTONIOU, V.; SKOPELITI, A. Measures and indicators of VGI quality: An overview. Proceedings of the ISPRS Annals of the Photogrammetry, Remote Sensing and Spatial Information Sciences, La Grande Motte, 2015. pp. 345-351.

BARRON, C.; NEIS, P.; ZIPF, A. A comprehensive framework for intrinsic OpenStreetMap quality analysis. Transactions in GIS, vol. 18, n.6, 2014. pp. 877-895

BRAVO, J. V. M.; SLUTER, R. C. O problema da qualidade de dados espaciais na era das Informações Geográficas Voluntárias. Boletim de Ciências Geodésicas, vol. 21, 2015. pp. 56-73.

BROVELLI, M. A.; ZAMBONI, G. A. New Method for the Assessment of Spatial Accuracy and Completeness of OpenStreetMap Building Footprints. International Journal of Geo-Information, vol. 7, n. 289, 2018.

BROWN, M.; SHARPLES, S.; HARDING, J.; PARKER, C. J.; BEARMAN, N.; MAGUIRE, M.; FORREST, D.; HAKLAY, M.; JACKSON, M. Usability of geographic information: Current challenges and future directions. Applied Ergonomics, vol. 44, 2013. pp. 855-865.

CARRASCO-LETELIER, L. Smartphones and tablet as scientific tools for georeferencing measurements. International Journal of Engineering Trends and Technology (IJETT), vol. 24, n. 2, 2015. pp. 82-86. 
CRUZ, D. T.; SANTOS, A. F. P. Controle de qualidade posicional do sistema rodoviário do Openstreetmap na região central De Viçosa-MG. Anais do VI Simpósio Brasileiro de Ciências Geodésicas e Tecnologias da Geoinformação, Recife, 2016.

DE TRÉ, G.; DE MOL, R.; HETEREN, S.; STAFLEU, J.; CHADEMENOS, V.; MISSIAEN, T.; KINT, L.; TERSELEER, N.; LANCKER, V. V. Data Quality Assessment in Volunteered Geographic Decision Support. In: BORDOGNA, G. (ed.); CARRARA, P. (ed.). Mobile Information Systems Leveraging Volunteered Geographic Information for Earth Observation. Springer International Publishing, 2017. pp. 173-192.

DEGROSSI, L. C.; ALBUQUERQUE, J. P.; SANTOS R. R.; ZIPF, A. A taxonomy of quality assessment methods for volunteered and crowdsourced geographic information. Transactions in GIS, vol. 22, 2018. pp. 542-560.

ELIAS, E. N. Qualidade de dados geoespaciais em plataforma de mapeamento colaborativo. Dissertação de mestrado. Universidade Federal da Bahia, Programa de Pós-Graduação em Engenharia Civil, Salvador, 2019. 197p.

ESMAIL, R.; NAESERI F.; ESMAIL, A. Quality assessment of Volunteered Geographic Information. American Journal for Geographic Information System, vol. 2, n. 2, 2013. pp.19-26.

FONTE, C. C.; ANTONIOU, V.; BASTIN, L.; ESTIMA, J.; ARSANJANI, J. J.; BAYAS, J-C. L.; SEE, L.; VATSEVA, R. Assessing VGI Data Quality. In: FOODY, G. (ed.); SEE, L. (ed.); FRITZ, S. (ed.); MOONEY, P. (ed.); OLTEANURAIMOND, A-M. (ed.); FONTE, C. C. (ed.); ANTONIOU, V (ed.). Mapping and the Citizen Sensor. Ubiquity Press, 2017. pp. 137-163.

GONÇALVES, D. A. R.; BORGES, R. M. A. Utilização de aplicativos móveis no ensino da Topografia. Evidência, vol. 12, n. 12, 2016. pp.147-158.

GRIRA, J.; BÉDARD, Y.; ROCHE, S. Spatial data uncertainty in the VGI world: Going from consumer to producer. Geomatica, vol. 64, n. 1, 2010. pp. 61-72.

GUEDES, E. M. P. Estudo de Técnica Híbrida de Localização de Estações Móveis Baseada em TDoA e AoA. Dissertação de mestrado. Instituto Militar de Engenharia, Programa de Pós-Graduação em Engenharia Elétrica, Rio de Janeiro, 2003. 119p. 
GUIMARÃES, A.; GRIVET, M. A. Radiolocalização de Terminais de Comunicações Móveis. Revista Científica Periódica - Telecomunicações, vol. 06, n. 01, 2003. pp. 1-17.

GOODCHILD, M. F. Citizens as sensors: the world of volunteered geography. Geojournal, vol 69, n. 4, 2007, pp. 211-221.

HAKLAY, M. How good is volunteered geographical information? A comparative study of OpenStreetMap and Ordnance Survey datasets. Environment and Planning B, vol. 37, n. 4, 2010. pp.682 -703.

HOFFMANN, G. P.; BORELLI, R. M. I. J.; SCHMIDT NANNI, A. O uso de geotecnologias livres: QGIS e EpiCollect no levantamento de dados em geociências. GeoFocus, n. 21, 2018. pp. 39-55.

HUNG, K.; KALANTARI, M.; RAJABIFARD, A. Methods for assessing the credibility of volunteered geographic information in flood response: A case study in Brisbane, Australia. Applied Geography, vol. 68, 2016. pp.37-47.

ISO 19157: Geographic Information - Data Quality. International Organization for Standardization, 2013.

LANGLEY, R. B. The mathematic of GPS. GPS World, vol. 2, n. 7, 1991. pp. 45-50.

LUCENA, Y. L. M. Utilização das redes celulares para posicionamento geodésico através do aparelho móvel. Trabalho de conclusão de curso. Universidade Federal de Pernambuco, Graduação em Engenharia Cartográfica e de Agrimensura, Recife, 2018, 81p.

MONICO, J. F. G.; PÓZ, A. P. D.; GALO, M.; SANTOS, M. C.; OLIVEIRA, L. C. Acurácia e precisão: revendo os conceitos de forma acurada. Boletim de Ciências Geodésicas, vol. 15, n. 3. 2009, pp. 469-483.

OLIVEIRA, G. A.; BRITO, P. L.; SANTOS, S. M. Mapeamento de riscos hidrológicos utilizando Informações Geográficas Voluntárias: Estudo de caso no município de Riachão do Jacuípe-Bahia. Revista Brasileira de Geografia Física, vol. 11, n. 01, 2018, pp. 297-311.

OLIVEIRA, G. A.; CIRILO, J. A.; RIBEIRO, A. A. S. Desenvolvimento de plataforma colaborativa para registro de inundações na escala intraurbana. Anais do XII Encontro Nacional de Águas Urbanas (ENAU), Maceió, 2018.

RÉQUIA, G. H. Desenvolvimento de Aplicativos CR-Campeiro Móbile: Caso de Teste: Sistema Operacional Android. Dissertação de Mestrado. 
Universidade Federal de Santa Maria, Programa de Pós-Graduação em Agronomia, Santa Maria, 2013. 70p.

SANTOS, A. P. Controle de qualidade cartográfica: metodologias para avaliação da acurácia posicional em dados espaciais. Tese de doutorado. Universidade Federal de Viçosa, Programa de Pós-Graduação em Engenharia Civil, Viçosa, 2015. 172 p.

SILVA, M. V.; NEVES, T. F. Mapeamento digital da cidade do Recife - PE em SIRGAS 2000. Anais do IV Simpósio Brasileiro de Ciências Geodésicas e Tecnologias da Geoinformação (SIMGEO), Recife, 2012. pp. 01-09.

TELEBRASIL, Associação Brasileira de Telecomunicações. Mapa de Estações Rádio Base (Antenas) no Brasil. Site <http://www.telebrasil.org.br/panorama-dosetor/mapa-de-erbs-antenas>, acessado em 07 de fevereiro de 2019.

TOMAŠTÍK, J.; TOMAŠTÍK, J.; SALOŇ, S.; PIROH, R. Horizontal accuracy and applicability of smartphone GNSS positioning in forests. Forestry: An International Journal of Forest Research, vol. 90, n. 2, 2017. pp. 187-198.

ZANDBERGEN P. A.; BARBEAU, S. J. Positional Accuracy of Assisted GPS Data from High-Sensitivity GPS-enabled Mobile Phones. Journal of Navigation, vol. 64, n. 3, 2011. pp. 381-399

ZANDBERGEN, P.A. Accuracy of iPhone locations: A comparison of assisted-GPS, WiFi and cellular positioning. Transactions in GIS, vol. 13, n. 1, 2009. pp. 525 .

ZEIMPEKIS, V.; GIAGLIS, G. M.; LEK, G. A taxonomy of indoor and outdoor positioning techniques for mobile location services. SIGecom Exchange, vol. 3, n.4, 2003. pp. 19-27. 